\title{
Extinction of gambling cue-reactivity: A pilot study in a problem gambling treatment setting
}

\author{
Ben J Riley ${ }^{*}$; Michael Baigent ${ }^{2}$; Sharon Harris²; Amii Larsen²; Tracey Nye ${ }^{2}$; Malcolm Battersby \\ ${ }^{1}$ Department of Health Sciences, Flinders University, South Australia \\ ${ }^{2}$ Department of Psychiatry, Flinders University, South Australia
}

\author{
*Corresponding Author(s): Ben J Riley \\ Department of Health Sciences, Flinders University, \\ Adelaide, Flinders Medical Centre, Block E2, The Flats, \\ Flinders Drive BEDFORD PARK SA 5042, South Australia \\ Tel: (61) 8 82046982; Email: ben.riley@.sa.gov.au
}

Received: Aug 02, 2018

Accepted: Nov 09, 2018

Published Online: Nov 14, 2018

Journal: Journal of Addiction and Recovery

Publisher: MedDocs Publishers LLC

Online edition: http://meddocsonline.org/

Copyright: (C) Raily BJ (2018). This Article is distributed under the terms of Creative Commons Attribution 4.0 International License

Keywords: Cue exposure; Problem gambling; Cue-reactivity; Urge; Pre-post design; Physiology

\section{Introduction}

\section{Cue-reactivity and gambling}

Gambling Disorder is classified by the Diagnostic and Statistical Manual of Mental Disorders (DSM5) as a non-substancerelated disorder within "Substance-Related and Addictive Disorders" [1]. It is a serious mental health condition associated with significantly harmful personal and social impacts such as occupational loss, family breakdown and suicide [2]. A conditioning model of addiction has been applied widely to substance use disorders [3]. The conditioning model maintains that any distinctive environment that is repeatedly associated with a drug

\begin{abstract}
Clinical interventions which focus on extinction learning have been shown to reduce craving and relapse in substance related and behavioural addictions. This paper reports a small pilot study with 20 problem gamblers referred for treatment by a local court diversion program. We investigated the use of portable heart rate monitors to measure the effectiveness of Cue Exposure Therapy (CET) in extinguishing gambling cue-reactivity. Cue-reactivity procedures consisted of a relaxation period followed by in-vivo exposure with response prevention in a gambling environment. Cue exposure therapy was manualised. Dependent measures comprised both self-report (gambling urge and problem gambling questionnaire) and physiologic measures (heart rate). Significant increases in heart rate were observed during in-vivo pre-CET but not post-CET ( $p<0.001)$. Following CET, significant reductions across all dependent variables were observed ( $p \leq 0.001$ ) with within-group effect sizes ranging between $r=-.55$ and -.61 . Overall, the results of this small pilot study support the feasibility and acceptability of the use of portable heart rate monitors to observe the extinction of gambling cue-reactivity. Portable heart rate monitors may provide a novel and useful tool for therapists and their problem gambling patients to monitor gambling cue-reactivity during treatment. Further research is needed to evaluate whether extinction of cue-reactivity can reduce problem gambling relapse.
\end{abstract}

effect, will eventually elicit a conditioned reaction called 'cue reactivity' [4]. Cue-reactivity has been demonstrated for example, by comparing reactivity to preferred versus non-preferred alcoholic beverages among alcoholics [5], and cigarettes versus neutral cues, for instance a glass of water among smokers [6]. A small but growing body of research suggests the cue-reactivity paradigm may be useful in understanding behavioural addictions such as problem gambling $[4,7]$. Sodano and Wulfert [8] compared cue-reactivity (involving video-tapes of gambling scenar- 
ios) among both active $(n=21)$ and abstinent $(n=21)$ problem gamblers and social gamblers $(n=21)$, and found no differences in gambling cue-elicited physiological responding (measured by Heart Rate [HR] in beats per minute [bpm]) between the groups, however the active problem gamblers displayed significantly higher subjective gambling urges. A recent study by McKeith, Rock and Clark [9] examined cue-reactivity among 38 poker machine gamblers. Self-reported gambling urges increased on presentation of gambling cues (3 minute videos) compared to neutral cues. In another study Potenza et al. [10] found male problem gamblers $(n=10)$ demonstrated higher gambling urges (measured by functional magnetic resonance imaging; fMRI) after viewing video tapes of gambling scenarios than controls $(n=$ 11). Similarly, a recent study by Limbrick-Oldfield et al. [11] used fMRI to examine gambling cue-reactivity, and found the gambling disorder group $(n=19)$ showed greater reactivity to personally tailored gambling cues (images) than controls $(n=19)$. Blanchard et al. [12] compared psychophysiological responses (skin resistance level, blood pressure and HR in bpm) among seven problem gamblers with seven age and gender matched controls in three settings: a standard stressor (a mental arithmetic task), gambling stimuli (individualised audio tapes of the gamblers' preferred method of gambling) and fear provoking stimuli (watching an individualised fear tape). All three groups displayed modest elevations in HR in response to the gambling tapes, while the active problem gamblers reported significantly greater subjective urges. The authors suggested that gambling cues used in an artificial laboratory setting (as opposed to a naturalistic in-vivo context) may only elicit a weak physiological response. This was also proposed by Anderson and Brown [13] who compared the cue-reactivity (measured by HR in bpm) of gamblers in an artificial casino and a real casino. Physiological responding was substantially and significantly greater in the invivo context, leading the authors to cast doubt on the validity of laboratory gambling as a measure of real gambling situations. In another study $(\mathrm{N}=48)$, imagery-based exposure (a written scenario designed to elicit craving to gamble) produced stronger self-reported gambling urges than photographic stimuli among university student gamblers $[14,15]$ examined cue-reactivity in 18 problem gamblers and found two thirds of the sample reported moderate to high gambling urges (self-report) during exposure to a casino environment. The authors recommended that cue exposure therapy be further researched as a potential tool in the treatment of problem gambling. Though the cue-reactivity paradigm has been well established for studying substance addictions, its application in the area of behavioural addictions is relatively new [7]. Based on the limited number of gambling related cue-reactivity studies reviewed, it appears to be a useful paradigm for investigating problem gambling, specifically, via observation of HR and in an in-vivo context. That being said, there is a dearth of literature concerning the use of cue-reactivity in the context of problem gambling treatment.

\section{Cue exposure therapy}

Cue exposure assumes that the cue-reactivity described above is classically conditioned. Non problem gamblers have been found to exhibit elevated HR in response to gambling behaviour [8]. Cue-reactivity is thus an Unconditioned Response (UR) to the stimulus of gambling behaviour itself. Neutral cues such as money and gambling venues become conditioned stimuli (or cues) for a gambling response. For the problem gambler, such cues as well as negative affect, can elicit an irresistible compulsive drive or urge to gamble [16]. Such compulsive drives are motivated by behaviour completion processes and if not fol- lowed through with, an aversive state of tension develops, producing an intensified level of compulsive drive [17]. Therefore, the urge to gamble is a negative state for the problem gambler and the gambling alleviates this state, and so is perpetuated by negative reinforcement. Battersby et al. [16] proposed gambling behaviour is maintained by the winning and losing sequences within this operant conditioning paradigm with a variable interval schedule of reinforcement. Based on conditioning theory, repeated exposure to the cue or CS without the associated response will result in extinction of the $C R$, if the exposure is prolonged until habituation occurs. That is, gambling cue-reactivity will be extinguished through repeated cue exposure with response prevention, as has been found for habituation of anxiety in Obsessive Compulsive Disorder (OCD). Cue exposure followed by response prevention, results in habituation of anxiety and improvement in OCD symptoms [18].

A handful of studies report the use of CET to treat problem gambling. Symes and Nicki [19] report two case studies using CET. In both cases gambling behaviour reduced substantially with abstinence maintained at one-month follow-up. The authors suggested that when subjects were thoroughly exposed to environmental, cognitive, behavioural and physical cues involved in salient gambling situations without obtaining a monetary reward, urges to gamble (measured by self-report) extinguished. Echeburua et al. [20] compared three therapeutic modalities for a group of problem gamblers $(N=64)$; (a) individual stimulus control with graded exposure and response prevention, (b) group cognitive restructuring and (c) combined therapy of $a+b$, and a fourth group involved a waitlist control. At 6 month follow-up there was no difference between the combined therapy and the control. At 12 month follow-up CET showed a higher success than the group or combined therapy. In another study ( $\mathrm{N}=69)$ Echeburua et al. [21] compared the effectiveness of exposure therapy combined with either group or individual relapse prevention along with a control group which received exposure therapy with no relapse prevention. All participants ceased gambling after the initial treatment with those that received exposure therapy plus one of the relapse prevention modalities showing greater improvement at 12 month follow-up than the control group. Oakes et al. [22] reported a case study using CET to treat a problem gambling disorder via video conferencing. The authors reported treatment gains maintained at 4 year follow-up. Tolchard et al. [23] reported a case study using single-session CET to treat a problem gambling disorder with treatment gains maintained at 6-month followup. Riley et al. [24] reported the significant reductions across all clinical measures for treatment completers ( $63 \%$ of the sample) in a problem gambling treatment service $(\mathrm{N}=551)$ using exposure therapy. A recent randomised control trial found problem gambling specific cognitive therapy and CET were both effective treatments at 6 month follow-up [25].

The limited literature to date suggests CET provides an effective mode of treatment for problem gambling, presumably by extinguishing gambling related cue-reactivity. However, no previous study has investigated the extinction of gambling related cue-reactivity via CET using physiologic measurement. The aim of this pilot study therefore, was to address this gap in the literature by examining the extinction of gambling related cue-reactivity following CET, using physiologic measurement. Further, we wanted to examine the feasibility of using portable HR monitors to measure extinction of cue-reactivity in a clinical setting, with the hope that the findings will help inform the development of eventual testing in a larger scale study. It was 
predicted that participants would display increased physiologic responding during exposure pre-CET, and that cue-elicited responding would be significantly decreased following CET.

\section{Methods}

\section{Participants}

Participants for this study comprised twenty individuals referred over an 18 month period for problem gambling treatment at a community based problem gambling treatment service in South Australia. Individuals were referred by the local Magistrates Court as part of an innovative court diversion program. All participants had pleaded guilty to committing an offence directly related to their gambling problem and agreed to participate in the CET program. Exclusion criteria for involvement in the court diversion program included the presence of an active substance abuse disorder (both illicit and non-prescribed medications) or other active mental health disorder such as psychosis. The first twenty-one individuals referred were originally considered eligible. Inclusion criteria for the present study were complete data on physiological measurements. One participant withdrew after the initial assessment due to a reluctance to engage in treatment and physiological measurement, opting instead to go through the standard court process. This left 20 problem gamblers ( 10 women) with an average age of 45 years $(S D=9.59)$. Fourteen $(70 \%)$ were of Australian, two Thai, three African and one South Pacific backgrounds. Sixteen (80\%) of participants gambled on electronic gaming/slot machines (one of these involved online slots) with the remaining four reporting wagering (three on horses/dogs; one on sports). The study was approved by the Southern Adelaide Clinical Human Research Ethics Committee, project number 402. 13, and all participants signed individual consent forms.

\section{Measures}

The following measures were collected at baseline and at completion of treatment (post-CET).

\section{Self-report measures}

The Victorian Gambling Screen Harm-to-Self sub-scale (VGSHS) is a 15-item simple and valid measure of PG [26]. It is a sub-scale of the Victorian Gambling Screen (VGS). The VGS-HS scores range from $0=$ no harm to self to $60=$ high harm to self. The VGS-HS has demonstrated high internal consistency among a clinical population of problem gamblers, with a Cronbach's alpha of 89 [26]. A score of 21 or greater indicates a problem gambling disorder.

The Gambling Urge Scale (GUS) is a 6-item single factor tool designed to measure gambling urges [27]. Items such as "It would be difficult to turn down a gamble this minute" and "all I want to do now is gamble" are answered on a seven-point Likert scale. Scores range from 0 to 47 with higher scores indicating higher urges. The scale has demonstrated good internal consistency with a Cronbach's alpha of .81 [27].

\section{Physiological measures}

HR was recorded using the Polar RC3-GPS with Polar H3 chest strap heart rate sensor. Polar monitors have been tested against standard clinical ECG equipment and found to be suitable for measuring HR $[28,29]$. HR data was sampled at 1 sample per second by the Polar H3 HR sensor chest strap which participants wore across their sternum under their clothing. Electrodes in the chest band transmitted HR data via a wireless signal through
Bluetooth $(2.4 \mathrm{GHz})$ technology to the RC3-GPS receiver. The receiver was worn on the wrist of the therapist/researcher. The Polar unit computed an average HR in bpm for each recording. HR data were uploaded from the RC3-GPS receiver to a desktop computer.

\section{Procedure}

Treatment: The CET treatment was delivered in individual face-to-face sessions and conducted according to a detailed manual that has been used previously in a randomised control trial $[25,30]$. Treatment comprised up to 12 weekly 60 -minute sessions, though clients may have received fewer or a greater number of sessions depending on the pace at which they moved through their graded cue exposure hierarchy. The treatment was delivered by three clinicians: a mental health social worker, psychiatric nurse and psychologist, all with postgraduate (masters) qualifications in CBT (http://www.flinders.edu.au/courses/ postgrad/cbt/) and each with more than seven years clinical experience delivering CET for gambling problems. All participants were given a standardised treatment rationale during their first session, along with an information booklet about the treatment and the physiologic measurement to read before their second session. During the second session a hierarchy of gambling cues was developed with each participant. This was a collaborative process between therapist and participant and utilised cues such as images and sounds of their preferred gambling activities including simulated slot machines, graded up to in-vivo exposure in a gambling environment. Clients were encouraged to perform CET homework regularly between sessions, as homework has been shown to predict outcome in exposure based treatment for problem gambling [32]. When performing graded exposure tasks, participants were instructed to remain focused on their urge until habituation had occurred. Once participants had extinguished their urge response from a particular cue, they moved to the next cue on their graded hierarchy.

\section{Self-report assessment}

Questionnaires were completed individually by participants in a quiet waiting room in the clinic at their first appointment and again after they had completed their CET program during their final session.

\section{Physiological measurement}

HR data were collected from participants before and after treatment. Pre-treatment data was collected at the second session. Participants were advised not to consume caffeine two hours prior or eat a meal 30 minutes prior to conducting physiologic measurement. Two HR measurements were collected at each time point: resting $H R$ and $H R$ in response to exposure to a $\mathrm{CS}$. The CS involved attending a relevant gambling environment, i.e. in-vivo. To record resting HR, participants were instructed to sit comfortably in an upholstered chair in a quiet room in the clinic and breathe normally. After allowing 5 minutes to habituate to the procedure, 5 minutes of continuous recording was performed. To record HR in the in-vivo setting, participants were accompanied by their therapist to a local gambling venue and instructed to sit comfortably on a chair in the gambling environment and breathe normally. Following a 5 minute period of quiet sitting they were instructed to observe and focus on the gambling activity paying special attention to their preferred CS (for example their favourite slot machine), while 5 minutes of continuous recording was collected. Pre and post-CET HR measurements were conducted at the same gambling environment 
for each participant. All therapists received training in conducting the HR testing procedure and followed a written protocol developed for the study.

\section{Statistical analysis strategy}

All analyses were conducted using SPSS 22.0 (IMB SPSS, New York, NY). An initial inspection of the data revealed two of the post-treatment dependent variables (VGS-HS; GUS) were positively skewed, consistent with a favourable outcome (data clustered around low values). Due to the non-normal distribution of these data and the small sample size, non-parametric tests were used to examine pre-post differences across variables [33]. Given this was a pilot study underpowered for traditional significance testing, we calculated the within-group effect sizes using methods described by Fritz, Morris and Richler [34]. In order to illustrate the magnitude and direction of cue-induced physiological changes, difference scores were calculated at both time points by subtracting the resting HR mean from the in-vivo exposure mean. Thus, positive difference scores indicate increases over baseline responding (resting HR) and negative difference scores indicate decreases. Difference scores (also referred to as change or gain scores) generally have considerably less variation than the scores from which they were created. Therefore, analyses using difference scores offer more statistical power than analyses conducted on post-test scores [35].

\section{Results}

The baseline means and SDs of all variables are presented in table 1. Baseline gambling harm-to-self scores (VGS-HS) were above the problem gambling cut off indicating clinically significant levels of problem gambling. Participants attended an average of 7.95 CET sessions (SD = 3.32).

\section{Effectiveness of CET outcome across dependent variables}

A Wilcoxon signed-ranks test revealed that the median postCET VGS-HS ranks were statistically significantly lower than the median post-CET VGS-HS ranks, median post-CET GUS ranks were statistically significantly lower than median pre-CET GUS ranks, and median post HR exposure ranks were statistically significantly lower than pre-CET HR ranks, with large within-group effect sizes [36], indicating the treatment was effective at reducing gambling related harm and gambling urges. Results of the Wilcoxon signed-ranks test are presented in Table 1.

\section{Physiological responding}

A further series of Wilcoxon signed-ranks tests were performed to examine physiologic cue-reactivity pre and posttreatment. At pre-CET, median HR ranks were statistically significantly higher during in-vivo exposure than median resting $\mathrm{HR}$ ranks, $\mathrm{Z}=-3.78, \mathrm{p}<0.001$, with a within-group effect size of $r=-.6$, indicating positive cue-reactivity pre-CET.

\section{Extinction of cue-elicited physiological responding}

Post-CET there were no significant differences between resting and in-vivo exposure median HR ranks, $p=0.68$, indicating extinction of cue-reactivity. Post-CET median difference score ranks were statistically significantly lower than pre-CET median ranks (table 2.).

\section{Correlation between self-report and physiological urge}

A Spearman's correlation revealed no significant associations between GUS and exposure HR, and no significant associations between GUS and HR difference scores $(p=0.59)$.

\section{Discussion}

In this pilot study of gambling cue-elicited physiologic responding, we observed that in-vivo gambling cues elicited a significant autonomic arousal response among problem gamblers, and that such responding was extinguished via CET. To our knowledge, this is the first study concerning the extinction of gambling cue-elicited responding among problem gamblers demonstrated by physiologic measurement. Furthermore, significant reductions in gambling harm-to-self and self-reported urge values were observed following CET, with all participants scoring below the problem gambling cut off. These preliminary data indicate CET may be a useful component in the treatment of problem gambling.

The average increase in HR we observed during pre-treatment in-vivo cue-exposure, was greater than that observed by Blanchard et al. [12] who used gambling cues in a laboratory setting, but substantially less than reported by Anderson and Brown [13] in an in-vivo setting, though it is important to note that participants in the latter study were actively gambling. The increase we found, of around seven bmp, is comparable to that of individuals with PTSD during exposure to traumatic imagery [37]. Further research is needed to understand the importance of psychophysiology and its role in Gambling Disorders. Not unlike other addictive and behavioural disorders such as alcohol addiction [38] and OCD [39], high rates of relapse have been observed among problem gamblers $[40,41]$. In a prospective cohort study of 158 problem gamblers, the compulsion or urge to gamble was found to be a significant predictor of both relapse, and continuing to gamble [31]. Thus, extinction of the urge to gamble appears to be an important factor in problem gambling recovery. Further research is needed to examine if extinction of physiologic cue-reactivity is related to lower relapse rates among problem gamblers. Although the GUS reduced significantly pre to post-CET, we found no association between self-reported gambling urge and HR during exposure. One possible reason for this other than the analyses lacking statistical power, is due to the single factor nature of the GUS. Young and Wohl [42] argue that in addition to a desire to gamble, craving to gamble also includes expectations of positive affect and relief from negative affect following gambling behaviour. That is, the urge to gamble is multi-dimensional. It may be that physiological arousal in response to gambling cues, is related more closely to anticipation of a positive affect rather than relief from negative affect. Blaszczynski and Nower's [43] widely accepted pathways model of problem gambling, proposes three distinct subgroups of gamblers; behaviourally conditioned, emotionally vulnerable, and antisocial, impulsivist problem gamblers. Future research could investigate cue-reactivity among these subgroups, which could have useful implications regarding the tailoring of treatment.

It is also possible that some gamblers may have had low levels of urge awareness. A number of participants in the present study scored low on their baseline self-report urge, and were surprised with their physiological response during pre-treatment exposure. The HR readings were a therapeutic demonstration to them of the presence and strength of an urge. There is some evidence that the trait of dispositional mindfulness, which reflects the inclination towards present moment awareness, is inversely related to $P G$ severity $[9,44,45]$. A future line of enquiry could examine the relationship between urge awareness and mindfulness. A third possibility is that as participants were part of a court diversion program, thus wanting to make a favourable 
impression on the court, they may have under reported their urges. Use of physiologic measurement may therefore provide a useful adjunct in this context. Limitations of this study include the relatively small sample size, which may have increased the possibility that statistically insignificant findings were due to insufficient statistical power. Measurements have not been analysed from the follow-up period so the sustainability of the changes is unknown. Given the well-known challenges inherent with the maintenance of new learning, such as renewal, spontaneous recovery and reinstatement [46], more research is required to investigate if gambling cue-elicited physiologic responding remains stable post extinction, and what strategies can be used to mitigate such threats to extinction training. The current study's sample comprised individuals who had engaged in criminal behaviour directly related to their gambling problem. They may be somewhat different to problem gamblers not facing criminal charges. Finally, we did not quantify homework engagement of participants in this pilot study. Engagement of
CET homework has been demonstrated to be significantly related to outcome for problem gambling, therefore a future study could quantify CET homework engagement and examine its relationship to extinction of cue-reactivity.

Future research needs to examine the extinction of cue-elicited physiologic responding via CET with non-criminal offending problem gambling populations. An additional line of enquiry could investigate recidivism rates among criminal offending problem gamblers who have extinguished their urge to gamble. Notwithstanding these limitations, this pilot study provides a first step in demonstrating the extinction of gambling related cue-reactivity using physiologic measurement. In addition, portable HR monitors may offer a novel and useful tool for problem gambling therapists and their patients to observe cue reactivity during the treatment process. The data and feasibility findings of this pilot study may provide an avenue for the further development of problem gambling interventions, outcome assessment and eventual testing in a larger scale study.

Tables

Table 1: Mean, SD, Mdn and IQR of study variables.

\begin{tabular}{|c|c|c|c|c|c|c|c|}
\hline Variable & Pre-CET & & & Post-CET & & & \\
\hline & Mean (SD) & Mdn & IQR & Mean (SD) & Mdn & IQR & $\begin{array}{c}\text { Wilcoxon signed ranks- } \\
\text { test Z }\end{array}$ \\
\hline VGS-HS & $26.05(16.86)$ & 35.00 & 33.00 & $3.55(4.45)$ & 1.50 & 5.80 & $-3.83^{*}$ \\
\hline GUS & $9.20(9.67)$ & 6.00 & 16.50 & $.2(.89)$ & 0 & 0 & $-3.41^{*}$ \\
\hline HR exposure & $86.9(10.32)$ & 87.50 & 13.80 & $79.25(10.85)$ & 79.00 & 19.00 & $-3.45^{*}$ \\
\hline
\end{tabular}

Note: * $\leq$ 0.001; VGH-HS, Victorian Gambling Screen Harm to Self Scale; GUS, Gambling Urge Scale; HR, heart rate.

Table 2: Changes in HR difference scores pre and post-CET.

\begin{tabular}{|c|c|c|c|}
\hline HR & Pre-CET & Post-CET & Wilcoxon signed-ranks test \\
\hline & Mean (SD) & Mean (SD \\
\hline Resting & $80(9.38)$ & $79.35(11.74)$ & $\mathrm{ns}$ \\
\hline $\begin{array}{c}\text { Exposure difference } \\
\text { score }\end{array}$ & $6.9(5.21)$ & $-.1(3.37)$ & $-3.88^{*}$ \\
\hline
\end{tabular}

Note: Note: HR: avg. Heart Rate in Beats Per Minute; ${ }^{*} \mathrm{p}<0.001$.

\section{References}

1. American Psychiatric Association. Diagnostic and statistical manual of mental disorders. (5th edition). Washington DC. 2013.

2. Productivity Commission. Gambling inquiry report. 2010; 1.

3. Carter BL, Tiffany ST. Meta-analysis of Cue-reactivity in Addiction Research. Addiction. 1999; 94: 327-340.

4. Rohsenow DJ, Childress AR, Monti PM, Niaura RS, Abrams DB. Cue Reactivity in Addictive Behaviors: Theoretical and treatment implications. International Journal of the Addictions. 1991; 25: 957-993.

5. Staiger PK, White JM. Cue reactivity in alcohol abusers: Stimulus specificity and extinction of the responses. Addictive Behaviors. 1991; 16: 211-221.

6. Carter BL, Tiffany ST. The cue-availability paradigm: The effects of cigarette availability on cue reactivity in smokers. Experimental and Clinical Psychopharmacology. 2001; 9: 183-190.

7. Katrin S, Antons S, Trorzke P, Brand M. Cue-reactivity in behavioral addictions: A meta-analysis and methodological considerations. Journal of Behavioral Addictions. 2018; 7; 227-238.

8. Sodano R, Wulfert E. Cue reactivity in active pathological, abstinent pathological, and regular gamblers. Journal of Gambling Studies. 2010; 26: 53-65.

9. McKeith CFA, Rock AJ, Clark GI. Trait mindfulness, problem-gambling severity, altered state of awareness and urge to gamble in poker-machine gamblers. Journal of Gambling Studies. 2017; 3: 617-632.

10. Potenza M, Steinberg M, Skudlarski P, Fulbright R, Lacadie C, et al. Gambling urges in pathological gambling: A Functional Magnetic Resonance Imaging study. Archives of General Psychiatry. 2003; 60: 828-836. 
11. Limbrick-Oldfield EH, Mick I, Cocks RE, McGonigle J, Sharman SP, et al. Neural substrates of cue reactivity and craving in gambling disorder. Translational Psychiatry. 2017; 7: 1-10.

12. Blanchard E, Wulfert E, Freidenberg B, Malta L. Psychophysiological Assessment of Compulsive Gamblers' Arousal to Gambling Cues: A Pilot Study. Applied Psychophysiology and Biofeedback. 2000; 25: 155-165.

13. Anderson G, Brown R. Real and laboratory gambling, sensationseeking and arousal. British Journal of Psychology. 1984; 75: 401-410.

14. Lisham A, McCarthy A, Rosenbery H. Assessing the impact of cue exposure on craving to gamble in university students. Journal of gambling studies. 2011; 28: 363-375.

15. Kushner MG, Abrams kB, Donahue C, Thuras P, Frost R, et al. Urge to gamble in problem gamblers exposed to a casino environment. Journal of Gambling Studies. 2007; 23: 121-132.

16. Battersby MW, Oakes JE, Tolchard B, Forbes A, Pols RG. Cognitive Behavioral Therapy for Problem Gamblers. In Masood Z, Blaszczynski A, Turner N. (Editors), In the pursuit of winning: Problem Gambling Theory, Research and Treatment. New York: Springer. 2008; 179-197.

17. McConaghy N, Armstrong M, Blaszczynski A, Allcock C. Controlled comparison of aversive therapy and imaginal desensitization in compulsive gambling. British Journal of Psychiatry. 1983; 142: 366-372.

18. Foa E. Cognitive behavioral therapy of obsessive-compulsive disorder. Dialogues Clinical Neuroscience. 2010; 12: 199-207.

19. Symes BA, Nicki RM. A Preliminary Consideration of cue-exposure, response-prevention treatment for pathological gambling behaviour: Two case studies. Journal of Gambling Studies. 1997; 13: $145-157$.

20. Echeburua E, Baez C, Fernandez-Montlalvo J. Comparative effectiveness of three therapeutic modalities in the psychological treatment of pathological gambling: Long-term outcome. Behavioural and Cognitive Psychotherapy. 1996; 24: 51-72.

21. Echeburua E, Fernandez-Montalvo J, Baez C. Relapse prevention in the treatment of slot-machine pathological gambling: Longterm outcome. Behaviour Therapy. 2000; 31: 351-364.

22. Oakes J, Battersby M, Pols R, Cromarty P. Exposure therapy for problem gambling via videoconferencing: A case report. Journal of Gambling Studies. 2007; 24: 107-118.

23. Tolchard B, Thomas L, Battersby M. Single-session exposure therapy for problem gambling: A single-case experimental design. Behaviour Change. 2006; 23: 148-155.

24. Riley B, Smith D, Oakes J. Exposure therapy for problem gambling in rural communities: A program model and early outcomes. Australian Journal of Rural Health. 2011; 19: 142-146.

25. Smith DP, Battersby MW, Harvey PW, Pols RG, Ladouceur R. Cognitive versus exposure therapy for problem gambling: Randomised controlled trial. Behaviour Research and Therapy. 2015; 69: 100-110.

26. Ben-Tovim D, Esterman A, Tolchard B, Battersby M. The Victorian Gambling Screen Project Report. Report prepared for the Victorian Casino and Gaming Authority, published by the Gambling Research Panel. Melbourne Victoria. 2001.

27. Namrata R, Tian O. The gambling urge scale: Development, confirmatory Factor validation and psychometric properties. Psychology of Addictive Behaviors. 2004; 18: 100-105.

28. Osanai H. Heart rate variability during a 24-hour period record- ed with a Polar heart rate monitor. Autonomic Neuroscience. 2010; 165: 439-448.

29. Vanderlei L, Silva R, Pastre C, Azevedo F, Godoy M. Comparison of the Polar S810i monitor and the EEG for the analysis of heart rate variability in the time and frequency domains. Brazilian Journal of Medical and Biological Research. 2008; 41: 854-859.

30. Battersby M, Oakes J, Redpath P, Harris S, Riley B. Statewide Gambling Therapy Service, Graded Exposure Therapy, a Treatment Manual for People with Gambling Problems. Flinders Human Behaviour and Health Research Unit - Flinders University. 2012.

31. Smith DP, Battersby MW, Pols RG, Harvey PW, Oakes JE, et al. Predictors of relapse in problem gambling: A prospective cohort study. Journal of Gambling Studies. 2015; 31: 299-313.

32. Riley BJ. The role of homework in exposure-based CBT outcome for problem gambling. International Gambling Studies. 2015; 15: 394-407.

33. Desu MM, Raghavarao D. Nonparametric statistical methods for complete and censored data: Chapman and Hall/CRC. 2004.

34. Fritz CO, Morris PE, Richler JJ. Effect size estimates: Current use, calculations, and interpretation. Journal of Experimental Psychology. 2012; 141: 2-18.

35. Salkind NJ. In Salkind NJ. (Editors), Encyclopedia of measurement and statistics. Thousand Oaks, California: Sage publications. 2007.

36. Cohen J. Statistical power analysis for the behavioral sciences (2nd edition). Hillsdale, NJ: Erlbaum. 1988.

37. Barkay G, Freedman N, Lester H, Louzoun Y, Sapoznikov D, et al. Brain activation and heart rate during script-driven traumatic imagery in PTSD: Preliminary findings. Psychiatry Research: Neuroimaging. 2012; 204: 155-160.

38. Moos R, Moos B. Rates and predictors of relapse after natural and treated remission from alcohol use disorders. Addiction. 2006; 101: 212-222.

39. Eisen JL, Sibrava J, Boisseau CL, Mancebo MC, Stout RL, et al. Five-year course of obsessive-compulsive disorder: Predictors of remission and relapse. Journal of Clinical Psychiatry. 2013; 74: 233-239.

40. Hodgins D, el-Guebaly N. Retrospective and prospective reports of precipitants to relapse in pathological gambling. Journal of Consulting and Clinical Psychology. 2004; 72: 72-80.

41. Hodgins DC, Currie SR, el-Guebaly N, Diskin KM. Does providing extended relapse prevention bibliotherapy to problem gamblers improve outcome? Journal of Gambling Studies. 2007; 23: 41-54.

42. Young MM, Wohl MJA. The Gambling Craving Scale: Psychometric validation and behavioral outcomes. Psychology of Addictive Behaviors. 2009; 23: 512-522.

43. Blaszczynski AP, Nower L. A pathways model of problem and pathological gambling. Addiction. 2002; 97: 487-499.

44. Lakey CE, Campbell WK, Brown KW, Goodie AS. Dispositional mindfulness as a predictor of the severity of gambling outcomes. Personality and Individual Differences. 2007; 43: 1698-1710.

45. Riley B. (2014). Experiential avoidance mediates the association between thought suppression and mindfulness with problem gambling. Journal of Gambling Studies. 2014; 30: 163-171.

46. Conklin C, Tiffany ST. Applying extinction research and theory to cue-exposure addiction treatments. Addiction. 2002; 97: 155167. 